\title{
Axonal Growth and Guidance Defects in Frizzled3 Knock-Out Mice: A Comparison of Diffusion Tensor Magnetic Resonance Imaging, Neurofilament Staining, and Genetically Directed Cell Labeling
}

\author{
Yanshu Wang, ${ }^{1 *}$ Jiangyang Zhang, ${ }^{2,3 *}$ Susumu Mori, ${ }^{2,4}$ and Jeremy Nathans ${ }^{1,5}$ \\ ${ }^{1}$ Department of Molecular Biology and Genetics, Howard Hughes Medical Institute, ${ }^{2}$ Department of Radiology, Division of Nuclear Magnetic Resonance \\ Research, ${ }^{3}$ Department of Biomedical Engineering, ${ }^{4}$ F. M. Kirby Research Center for Functional Brain Imaging, Kennedy Krieger Institute, and \\ ${ }^{5}$ Departments of Neuroscience and Ophthalmology, Johns Hopkins University School of Medicine, Baltimore, Maryland 21205
}

\begin{abstract}
Previous work has identified axonal outgrowth and/or guidance defects in the brain and spinal cord of prenatal Frizzled3 (Fz3) ${ }^{-1-}$ mice. To systematically explore the axonal defects in $\mathrm{Fz}^{-/-}$mice and to compare techniques for the global assessment of axon tracts in the developing mouse, we have analyzed wild-type and $\mathrm{Fz}^{-/-}$brains using (1) diffusion tensor magnetic resonance imaging ( $\mu$ DTI), (2) neurofilament staining, and (3) two genetically directed neuronal labeling methods. Confirming and extending the previous work of Wang et al. (2002), we find that the following structures/tracts are absent or greatly reduced in the $\mathrm{Fz} 3^{-/-}$brain: the anterior commissure, cerebral peduncle (corticospinal tract), corpus callosum, fornix, internal capsule (thalamocortical and corticothalamic tracts), stria medullaris, stria terminalis, and hippocampal commissure. An aberrant U-shaped fiber bundle immediately caudal to the optic tract connects the left and right sides of the $\mathrm{Fz}^{-/-}$thalamus and likely represents a default pathway for thalamic axons that failed to enter the internal capsule. At embryonic day 18, labeling of cortical pyramidal cells with a yellow fluorescent protein reporter reveals widespread fragmentation of axons with no apparent loss of pyramidal cell bodies. Fragmentation likely represents one stage in the process that normally eliminates stalled or mistargeted axons. This work demonstrates the usefulness of $\mu$ DTI and genetically directed neuronal labeling for the analysis of nervous system defects in the mouse.
\end{abstract}

Key words: brain development; Frizzled3; axon guidance; fiber tracts; cre recombinase; YFP; knock-out mice; magnetic resonance imaging; diffusion tensor; axonal fragmentation

\section{Introduction}

It has long been appreciated that the brain exhibits a large number of precise and long-range axonal connections, and, for more than a century, the analysis of these axon tracts has been a major preoccupation of neuroanatomists and neurologists. The first methods for systematically mapping the trajectories of axon tracts were based on the selective staining of degenerating axons and their associated myelin sheaths after a localized anatomic lesion (Nauta and Feirtag, 1986). The development of anterograde, retrograde, and bidirectional tracers (including horse radish peroxidase, fluorescent dextrans and microspheres, and carbocyanine dyes) has greatly refined this analysis and, in the case of

Received Aug. 2, 2005; revised Nov. 9, 2005; accepted Nov. 10, 2005.

This work was supported by the National Institutes of Health Grants R01AG20012 and R01EB003543 (J.Z., S.M.) and the Howard Hughes Medical Institute (Y.W., J.N.). We thank the following: Tudor Badea for advice; John Williams for genotyping support; Guoping Feng, Corrine Lobe, Andras Nagy, and Joshua Sanes for making the YFP and ZAP mice freely available; and Chunqiao Liu and Amir Rattner for helpful comments on this manuscript.

*Y.W. and J.Z. contributed equally to this work.

Correspondence should be addressed to Dr. Jeremy Nathans, 805 PCTB, 725 North Wolfe Street, Johns Hopkins University School of Medicine, Baltimore, MD 21205. E-mail: jnathans@jhmi.edu.

DOI:10.1523/JNEUROSCI.3221-05.2006

Copyright $\odot 2006$ Society for Neuroscience $\quad$ 0270-6474/06/260355-10\$15.00/0 carbocyanine dyes, permitted axon tracing in postmortem tissue (Haugland, 2002). Each of these methods relies on the localized placement of an axonal tracer, resulting in the visualization of one, or at most a few, axon tracts. They do not, therefore, lend themselves to large-scale or high-throughput surveys of axonal connections.

With the development of gene targeting and large-scale chemical mutagenesis screens in the mouse, there is an increasing need for methods that can be used to systematically and efficiently survey axon tracts within the developing and adult mouse brain. At present, axon tracts in the mouse brain are typically surveyed by immunolocalizing neurofilament proteins in serial thin sections obtained from either frozen or wax-embedded tissue. Although this approach permits the accurate visualization of major axon tracts, it does not lend itself to three-dimensional (3D) reconstruction because of section-to-section variability and the difficulty in recovering dozens of sections at uniform intervals. As a method for axonal tracing, neurofilament localization also suffers from a high density of labeling in many brain regions, which can obscure the different trajectories of closely apposed or interdigitating tracts.

In the present study, we compare neurofilament staining with 

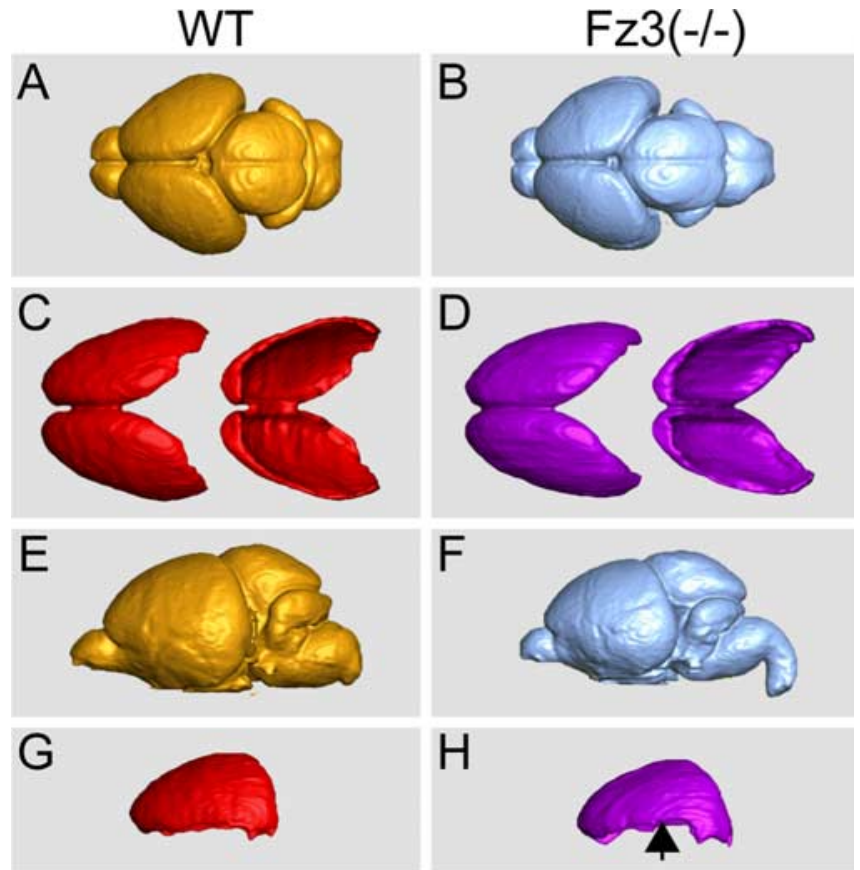

Superposition of WT and
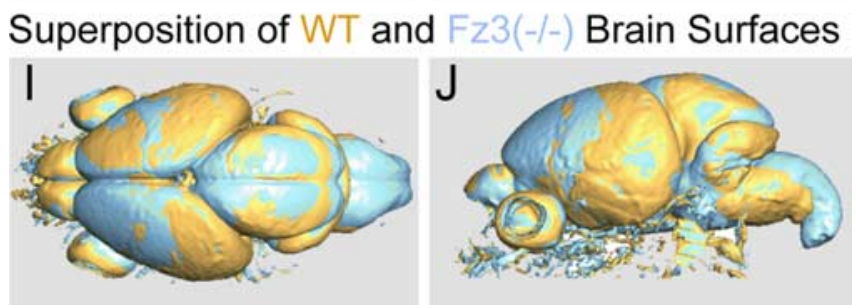

Figure 1. Surface morphology of WT and $F z 3^{-/-}$brains and cerebral cortices at E18 determined by MRI. $\boldsymbol{A}, \boldsymbol{B}, \boldsymbol{I}$, Dorsal view; $\boldsymbol{E}, \boldsymbol{F}, \boldsymbol{J}$, lateral view. $\boldsymbol{C}, \boldsymbol{D}$, Dorsal (left) and ventral (right) views of the cerebral cortex; $\boldsymbol{G}, \boldsymbol{H}$, lateral view of the cerebral cortex. $\boldsymbol{I}, \boldsymbol{J}$, A superposition of WT and $\mathrm{Fz} 3^{-/-}$surfaces. The vertical arrow in $\boldsymbol{H}$ indicates the more dorsal location of the lateral edge of the neocortex in the $\mathrm{Fz}^{-1-}$ brain.

three newer methods for analyzing axonal trajectories. The first of these methods is magnetic resonance (MR) diffusion tensor microimaging ( $\mu$ DTI) (Jacobs et al., 1999; Mori et al., 2001). Unlike standard MR imaging (MRI) based on T1 and T2 relaxation properties of water molecules, $\mu$ DTI reveals the locations and orientations of axon tracts by distinguishing regions in which water (the major repository of protons) shows a spatial anisotropy in its diffusion. $\mu$ DTI has a number of advantages for quantitative analysis: the data are free of sectioning artifacts and can be viewed in any plane, the shape and volume of various anatomic structures are readily calculated, and different datasets can be compared quantitatively.

The remaining two methods used here are based on the expression of transgenic reporters in subsets of neurons. One method uses a yellow fluorescent protein (YFP) transgene driven by the Thyl promotor (Feng et al., 2000). The second method uses pharmacologic activation of site-specific DNA recombination in transgenic mice to produce a sparse population of cells expressing human placental alka- line phosphatase (AP) (the CreER;ZAP system) (Badea et al., 2003).

We have chosen to compare these methods as part of a comprehensive analysis of the phenotype of Frizzled $3(\mathrm{Fz} 3)^{-1-}$ mice. Frizzled proteins are cell surface receptors that participate in a wide variety of developmental processes, including cerebellar development and retinal vascularization [Fz4 (Wang et al., 2001)], hair patterning [Fz6 (Guo et al., 2004)], and cell survival in the hippocampus [Fz9 (Zhao et al., 2005)]. Fz3 is widely expressed in the developing nervous system, and our initial analysis of $\mathrm{Fz}^{-/-}$ animals revealed an absence or severe reduction in the size of several major axon tracts in the developing forebrain and a failure of sensory axons in the spinal cord to grow rostrally after crossing the midline (Wang et al., 2002; Lyuksyutova et al., 2003). These data implicated $\mathrm{Fz} 3$ in axonal growth and guidance and suggested that additional defects might be revealed by a systematic analysis of axon tracts throughout the developing nervous system. The observations presented here define these additional defects, and they reveal a widespread fragmentation of CNS axons that have failed to find their targets.

\section{Materials and Methods}

Mouse genetics. Because $\mathrm{Fz}^{-/-}$mice die shortly after birth, $\mathrm{Fz} 3$ mutant mice were maintained in the heterozygous state. $F 3^{-1-}$ embryos, recognized by their curled tails (Wang et al., 2002), and control $\mathrm{Fz}^{+/-}$and $\mathrm{Fz}^{+/+}$littermates, recognized by their straight tails, were harvested at the indicated gestational ages from timed matings. To mark cells with AP, $\mathrm{Fz}^{+/-}$; ROSA26-creER/ROSA26-creER males or females were mated to $\mathrm{Fz}^{+/-} ; \mathrm{ZAP} /+$ females or males, respectively. We note that the ZAP transgene cannot be maintained in the homozygous state (Lobe et al., 1999); with the optimal mating strategy, only one in eight embryos is

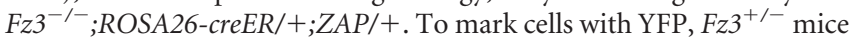
carrying either one or two copies of the Thyl-YFP transgene (line $\mathrm{H}$ ) (Feng et al., 2000) were mated.

Neurofilament staining. For whole-mount neurofilament staining, embryonic day 12 (E12) embryos were fixed for $4 \mathrm{~h}$ in PBS with $4 \%$ paraformaldehyde (PFA) and stained with anti-neurofilament mouse monoclonal antibody $2 \mathrm{H} 3$ (Developmental Studies Hybridoma Bank, Iowa City, IA) using a 1:50 dilution of conditioned medium in PBS with $0.3 \%$ Triton X-100. For staining of paraffin sections, embryos were immersed in Carnoy's fixative, embedded in paraffin, processed as described by Wang et al. (2002), and stained with rabbit anti-neurofilament $\mathrm{M}$ antibodies (AB1987; Chemicon, Temecula, CA).

CreER;ZAP labeling. Pregnant females were given a single intraperitoneal injection of $200 \mu \mathrm{g}$ of 4-hydroxytamoxafen (4HT) in sunflower seed
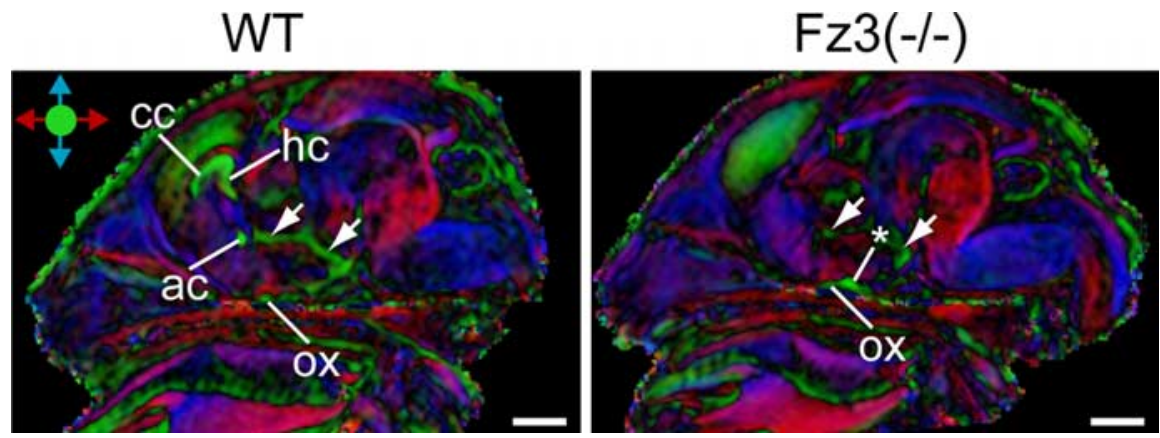

Figure 2. Midsagittal $\mu \mathrm{DTI}$ images of WT and $\mathrm{Fz} 3^{-/-}$brains at E18. Anterior is to the left. The $\mathrm{Fz} 3^{-/-}$brain is missing the corpus callosum (cc), anterior commissure (ac), and hippocampal commissure (hc). The optic chiasm (ox) is present in both WT and $\mathrm{Fz}^{-1-}$ brains. The region marked by an asterisk immediately posterior to the optic chiasm in the $\mathrm{Fz} 3^{-/-}$brain is the midsagittal part of a novel U-shaped fiber bundle within the ventral thalamus. The arrows indicate oriented cells in the wall of the third ventricle. The key in the top left illustrates the false color code for the directions of maximal diffusion with respect to the image plane: horizontal is represented by blue, vertical is represented by red, and perpendicular to the image plane is represented by green. An analogous false color code applies to each of the $\mu$ DTI images in later figures. Scale bars, $1 \mathrm{~mm}$. 

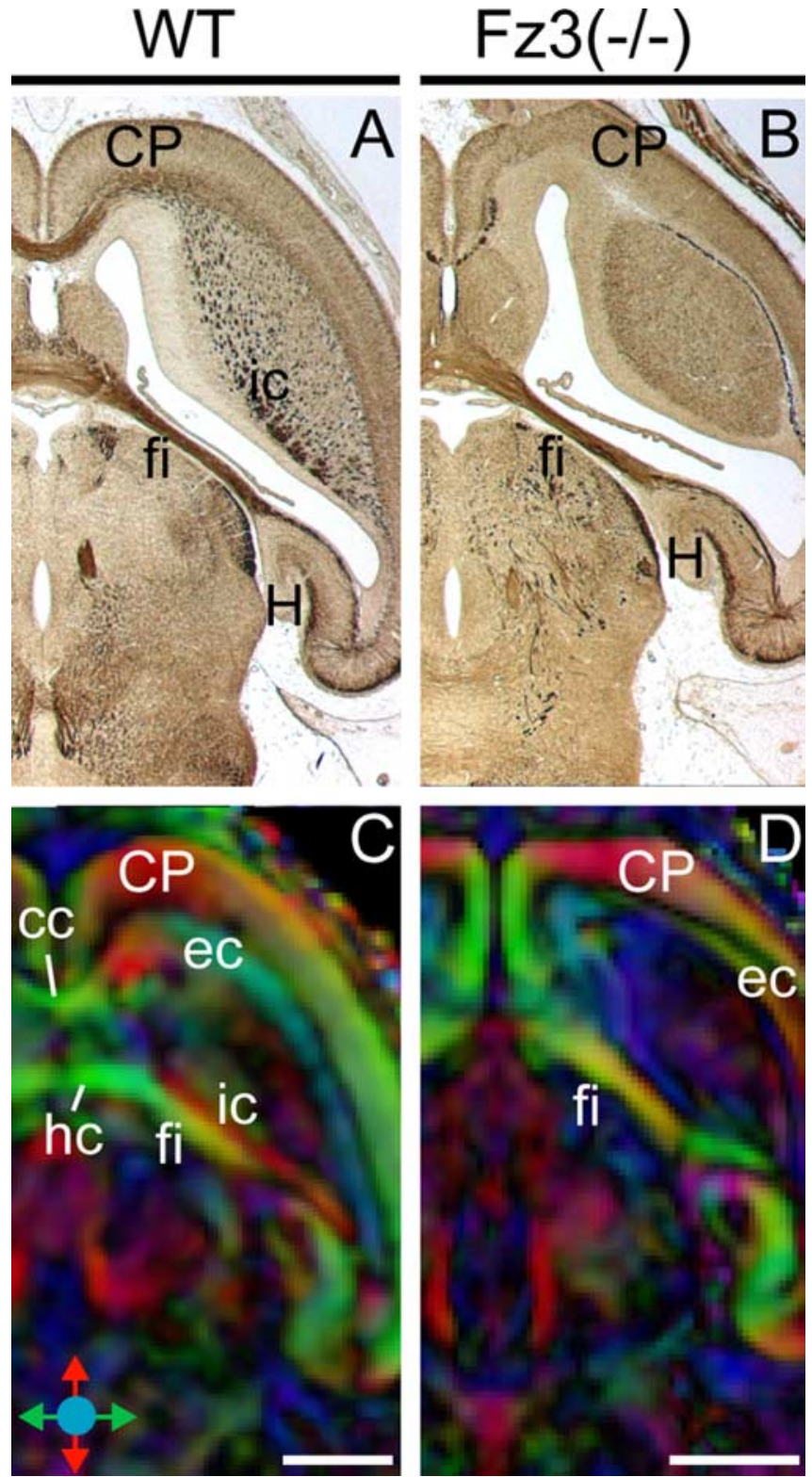

Figure 3. Comparison of neurofilament immunostaining and $\mu \mathrm{DTI}$ of WT and $\mathrm{Fz}_{2}{ }^{-/}$ brains in horizontal section at E18. The midline is adjacent to the left border of each image. In the $\mathrm{Fz}_{3}{ }^{-/-}$brain, the density of subcortical fibers and the thickness of the corpus callosum are markedly reduced, and the hippocampus and fimbria appear approximately normal but the number of fibers crossing the midline at the hippocampal commissure is reduced. The key in the bottom left of $\boldsymbol{C}$ illustrates the false color code for oriented diffusion for $\boldsymbol{C}$ and $\boldsymbol{D}$. cc, Corpus callosum; CP, cortical plate; ec, external capsule; fi, fimbriae; $\mathrm{H}$, hippocampus; hc, hippocampal commissure; ic internal capsule. Scale bars, $1 \mathrm{~mm}$.

oil between 10 and $13 \mathrm{~d}$ of gestation. Tissue processing and AP histochemistry were performed essentially as described by Badea et al. (2003) using $300 \mu \mathrm{m}$ vibratome sections of immersion-fixed brains. A total of eight pairs of $\mathrm{Fz}^{-/-}$and wild-type (WT) littermates were analyzed by AP staining.

Thy1-YFP tissues. E18 brains were dissected, immersion fixed in cold PBS with $2 \%$ PFA for $2 \mathrm{~h}$ at $4^{\circ} \mathrm{C}$, embedded in 3\% low-melting point agarose in PBS, and sectioned at $200 \mu \mathrm{m}$ on a vibratome. The sections were imaged with a Zeiss (Oberkochen, Germany) LSM510 confocal microscope.

MRI. Fz3 ${ }^{-1-}$ and WT heads were harvested at either E14 (one WT and two $\mathrm{Fz}^{-{ }^{-1-}}$ littermates) or E18 (two pairs of $\mathrm{Fz}^{-{ }^{-1}}$ and WT littermates). The skin was removed, and the heads were immersion fixed for $1 \mathrm{~d}$ in PBS with $4 \% \mathrm{PFA}$ at $4^{\circ} \mathrm{C}$. Imaging was performed using an 11.7 Tesla spectrometer and a Micro5.0 gradient system with a maximum gradient strength of 300 Gauss/cm (Bruker BioSpin, Billerica, MA). A 10-mmdiameter saddle coil was used as the radio frequency signal transmitter and receiver. Images were acquired using a 3D fast echo sequence with navigator-echo phase correction scheme (Mori and van Zijl, 1998), with a repetition time of $900 \mathrm{~ms}$, an echo time of $26 \mathrm{~ms}$, four signal average, and an echo train length of four. The navigator-echo phase correction scheme removes artifacts attributable to imperfections in the radio frequency pulse, gradient stability, and gradient echo currents. The native imaging resolution was $\sim 0.08 \times 0.08 \times 0.08 \mathrm{~mm}$. The spectral data were apodized by a $10 \%$ trapezoidal window function and then zero filled to double their sizes. Eight diffusion-weighted images were acquired, with two images of minimum $b$ value $\left(150 \mathrm{~s} / \mathrm{mm}^{2}\right)$ and the rest with maximum $b$ value $\left(\sim 1000-1200 \mathrm{~s} / \mathrm{mm}^{2}\right)$. Diffusion sensitizing gradients were applied along six different orientations: $[0.707,0.707,0],[0.707,0$, 0.707], [0, 0.707, 0.707], [-0.707, 0.707, 0], [0.707, 0, -0.707], and [0, $-0.707,0.707]$. The total imaging time was $\sim 15 \mathrm{~h}$.

The diffusion tensor was calculated using a log-linear fitting method (Basser et al., 1994). For the quantification of anisotropy, a linear measure [chemiluminescence (CL)] was used (Westin et al., 2002). Isotropicdiffusion weighted images (iDWIs) were calculated as the sum of the six diffusion-weighted images. Color map images were generated by combining the images of the primary eigenvector and the CL into red-greenblue images. Red was assigned to the caudal-to-rostral axis, green to the medial-to-lateral axis, and blue to the dorsal-to-ventral axis. The intensity was proportional to the CL. For E18, iDWIs of one subject were selected as the template, and the iDWIs of the other subjects were registered to the template using the six-parameter rigid transformation capability included in the automated image registration software (Woods et al., 1998). The same procedure was performed on images from E14 samples. The brain surfaces were extracted from iDWIs with a carefully selected intensity threshold. Brain surfaces and surfaces of manually segmented cortical plates were rendered in 3D using Amira (Mercury Computer Systems, Chelmsford, MA) for visual examination.

\section{Results}

\section{Morphology of the $\mathrm{Fz}^{-/-}$brain}

As an initial step in characterizing the Fz3 mutant phenotype with MRI, the surface morphologies of representative WT and $F z 3^{-/-}$ brains were obtained from scans of the intact cranium (Fig. 1). (To minimize biological variability, all comparisons of WT and $\mathrm{Fz}^{-/-}$samples described here used littermates.) As seen from a superposition of the two surface images, WT and $\mathrm{Fz}^{-/-}$brains at E18 are nearly identical in both size and shape (Fig. $1 I, J$ ). The surface morphologies of $\mathrm{Fz}^{-/-}$and WT cerebral cortices were also determined and found to differ principally by the more dorsal location of the lateral border of the neocortex, the rhinal sulcus, in the $\mathrm{Fz}^{-/-}$brain (Fig. $1 \mathrm{C}, \mathrm{D}, \mathrm{G}, \mathrm{H}$ ).

\section{Fiber tract defects in the $\mathrm{Fz}^{-/-}$brain}

To systematically assess fiber tract defects in the $\mathrm{Fz}^{-/-}$brain, complete serial $\mu \mathrm{DTI}$ images of E18 WT and $\mathrm{Fz}^{-/-}$brains were visually examined side-by-side in coronal, horizontal, and sagittal section. In the midsagittal plane, the $F z 3^{-/-}$brain shows little or no evidence of the corpus callosum and the hippocampal and anterior commissures (Fig. 2). Because the spatial resolution of the $\mu \mathrm{DTI}$ images is $\sim 80 \mu \mathrm{m}$, the failure to observe a particular tract with $\mu$ DTI does not rule out the presence of a small number of fibers. For example, we typically observe a small and variable number of fibers in the corpus callosum in the $F z 3^{-/-}$brain with neurofilament staining (see below).

For the purpose of comparing $\mu \mathrm{DTI}$ and anti-neurofilament immunostaining in fiber tract analyses, Figures $3-5,7$, and 8 show $\mu$ DTI image planes that have been matched to a series of neurofilament-stained paraffin sections. The horizontal sections 

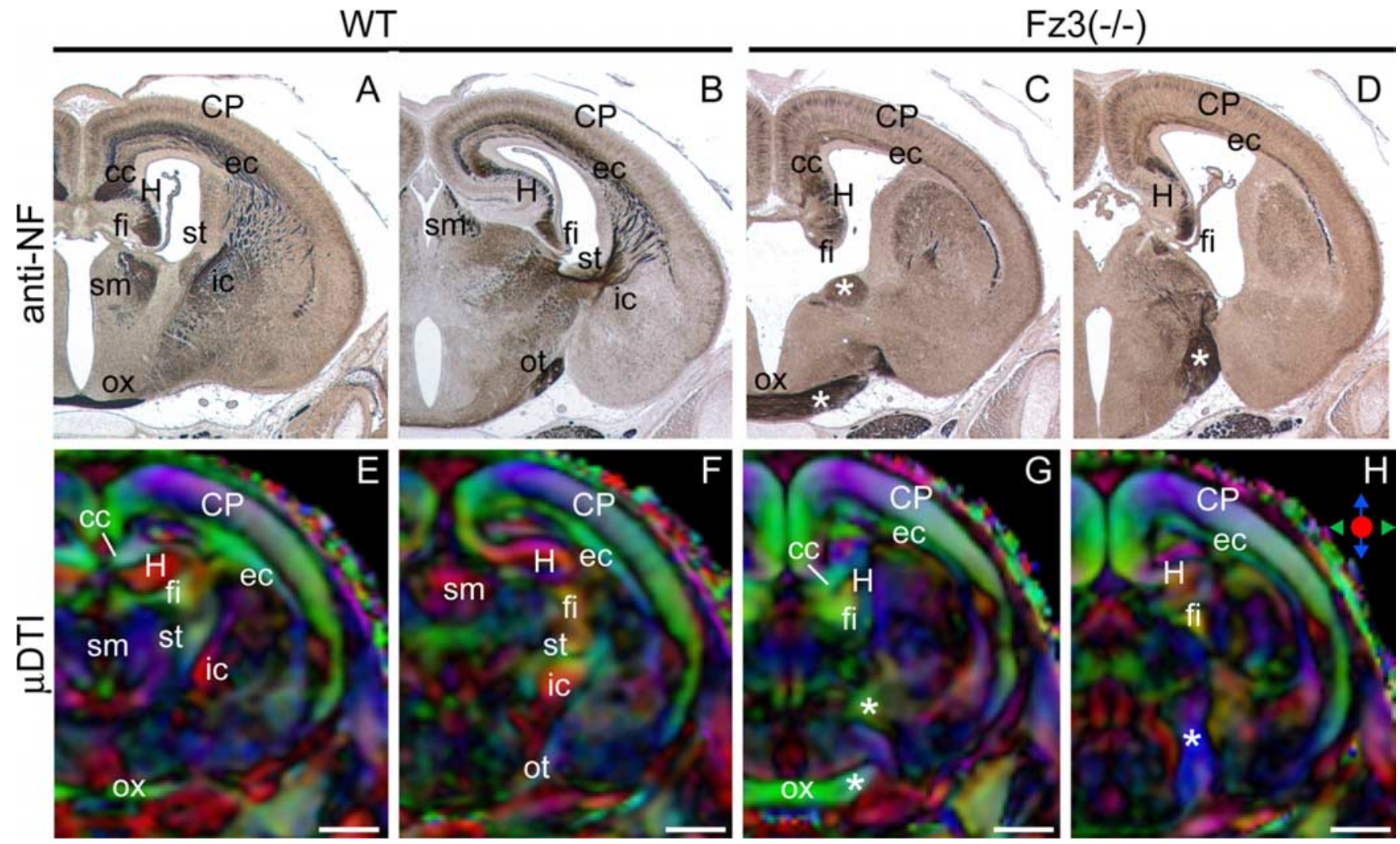

Figure 4. Comparison of neurofilament (NF) immunostaining and $\mu \mathrm{DTI}$ of WT and $\mathrm{Fz} 3^{-/-}$brains in coronal section at E18. Sections are at the level of the optic chiasm (left member of each pair of images) and $\sim 300 \mu \mathrm{m}$ posterior (right member of each pair of images). The midline is adjacent to the left border of each image. In the $F 3^{-/-}$brain, the internal capsule is missing, and a novel U-shaped fiber bundle marked by asterisks links the dorsolateral regions of the left and right sides of the thalamus. The key in the top right of $\boldsymbol{H}$ shows the false color code for oriented diffusion in $\boldsymbol{E}$-H. cc, Corpus callosum; CP, cortical plate; ec, external capsule; fi, fimbria; H, hippocampus; ic, internal capsule; ot, optic tract; ox, optic chiasm; st, stria terminalis; sm, stria medullaris. Scale bars, $1 \mathrm{~mm}$.

in Figure 3, taken at the level of the hippocampal commissure at E18, show nearly equivalent fimbria in the WT and mutant brains, but, in the $\mathrm{Fz}^{-{ }^{--}}$brain, most of these fibers fail to cross the midline. With neurofilament staining, the $F z 3^{-/-}$brain also shows a near absence of fibers at the midline of the corpus callosum, consistent with the midsagittal and horizontal views obtained by $\mu$ DTI (Figs. 2, 3). In the Fz3 $3^{-/-}$cortex at E18, $\mu$ DTI reveals the normal pattern of radial polarity, consistent with previous histologic data showing that cell proliferation and migration in the cortex are unaffected by loss of $F z 3$ (Wang et al., 2002). Interestingly, in the $\mathrm{Fz}^{-/-}$thalamus, the complex pattern of nerve fibers is distinctively different from the WT (Fig. 3), a point that will be further addressed below.

Coronal sections at E18 illustrate the previously described and most dramatic fiber tract defect associated with loss of Fz3: the complete or nearly complete absence of thalamocortical connectivity via the internal capsule (Fig. 4) (Wang et al., 2002). Additionally, both the anti-neurofilament-stained sections and the $\mu$ DTI images reveal the loss of the stria medullaris and a previously unreported $U$-shaped fiber bundle that is unique to the $\mathrm{Fz}^{-1-}$ brain and that courses adjacent to the optic tract along the ventral and lateral edges of the thalamus (Fig. $4 C, D, G, H$, asterisks). The intrathalamic trajectory of this U-shaped fiber tract suggests that it is composed of thalamic axons that would normally have entered the internal capsule but that, in the $\mathrm{Fz}^{-/-}$ brain, have been rerouted to the contralateral thalamus. In horizontal sections, the $\mathrm{U}$-shaped fiber tract is seen to reside immediately posterior to the optic tract (Fig. 5).
In comparing $\mu \mathrm{DTI}$ and neurofilament-stained brains from mice of matched genotypes, the latter were seen to have substantially larger ventricles (Fig. 4). We suspect that this difference derives from differences in tissue fixation: the brains processed for immunohistochemistry were immersion fixed in cold acetic acid/ethanol/chloroform (Carnoy's fixative) and then dehydrated in ethanol, whereas the brains processed for $\mu$ DTI were immersion fixed in PBS with paraformaldehyde. In the former procedure, dehydration would be predicted to produce tissue shrinkage and ventricular enlargement, whereas the latter procedure would likely be associated with tissue swelling and ventricular narrowing. Because of these uncertainties in fixation effects on tissue volume, we have not attempted to quantitate ventricular volumes. A challenge for the future will be to develop an MRI-compatible immersion fixative for mouse embryos that is free of volume distortion.

To visualize the spatial relationships among major fiber tracts within and adjacent to the thalamus, voxels from the $\mu$ DTI dataset corresponding to the fibers of the internal capsule, optic tract, and stria terminalis in the WT brain and to the U-shaped thalamic fibers and the optic tract in the $\mathrm{Fz}^{-1-}$ brain were color coded and visualized in horizontal, sagittal, and coronal views (Fig. 6). [The stria terminalis is not identifiable in the $\mathrm{Fz}^{-/-}$ brain.] This analysis illustrates the extent of the adjacent trajectories of the optic tract and the U-shaped fiber bundle in the $\mathrm{Fz}^{-1-}$ brain. Despite the substantial rerouting of most thalamic fibers in the $\mathrm{Fz}^{-/-}$brain, the trajectory of the optic tract is minimally if at all affected. 


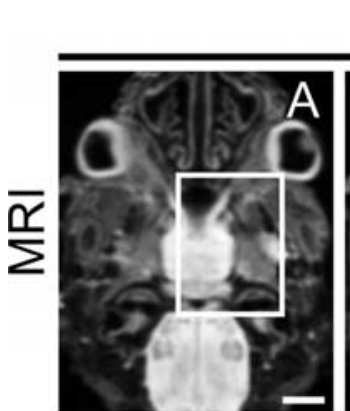

Fz3(-/-)
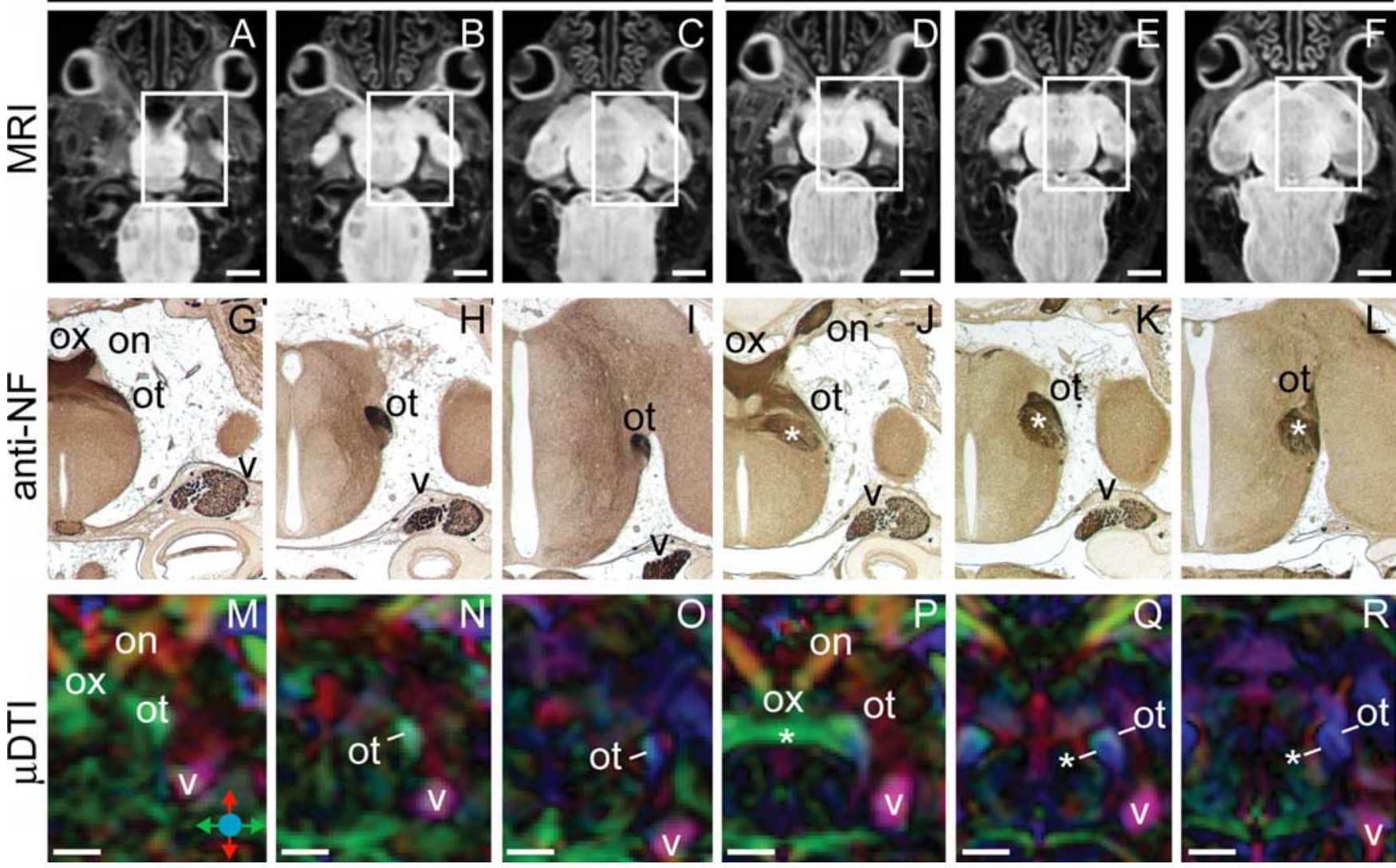

Figure 5. Neurofilament (NF) immunostaining and $\mu \mathrm{DTI}$ of the ventral thalamus at E18 showing the trajectory of the U-shaped fiber bundle in the $F z 3^{-/-}$brain in horizontal section. $\boldsymbol{A - F}$, Conventional MRI of the head with the anterior at the top. $G-L$, Neurofilament staining of the ventral thalamus and surrounding structures; $M-R, \mu D T I$ of the ventral thalamus and surrounding structures. The locations of the individual $\mu \mathrm{DTl}$ images $(\boldsymbol{M}-\boldsymbol{R})$ are shown by the rectangular insets in $\boldsymbol{A}-\boldsymbol{F}$. In $\boldsymbol{G}-\boldsymbol{R}$, the midline is adjacent to the left border of each image. The $U$-shaped fiber bundle, marked by an asterisk, courses caudal to the optic tract. The key in the bottom right of $\boldsymbol{M}$ shows the false color code for oriented diffusion in $\boldsymbol{M}-\boldsymbol{R}$. on, 0 ptic nerve; ot, optic tract; ox, optic chiasm; $V$, trigeminal nucleus. Scale bars: $\boldsymbol{A}-\boldsymbol{F}, 1 \mathrm{~mm} ; \boldsymbol{M}-\boldsymbol{R}, 0.5 \mathrm{~mm}$.

Comparison of $\mu \mathrm{DTI}$ and neurofilament staining in the analysis of the E14 brain

By DiI labeling, defects in thalamocortical and corticothalamic fiber tracts are observed in the $F z 3^{-1-}$ brain as early as E13 (Wang et al., 2002). To compare the utility of $\mu$ DTI and neurofilament staining for phenotypic characterization of the developing mouse brain before E18, we compared WT and $\mathrm{Fz}^{-1-}$ brains at E14 (Fig. 7). At this stage, the brain is $\sim 3 \mathrm{~mm}$ in width; as noted in Materials and Methods, the resolution of $\mu \mathrm{DTI}$ is $\sim 80 \mu \mathrm{m}$. As seen in both coronal (Fig. $7 G-L$ ) and horizontal (Fig. $7 R, T$ ) planes, $\mu$ DTI readily reveals the absence of the internal capsule and the presence of the U-shaped thalamic fibers in the $\mathrm{Fz}^{-1-}$ brain at E14. At this age, the cerebral peduncle is also missing in the $F z 3^{-/-}$brain, but the optic chiasm and the fasciculus retroflexus are present. Neither the corpus callosum nor the anterior commissure are sufficiently developed to be seen by $\mu \mathrm{DTI}$ at E14 in either WT or $F z 3^{-1-}$ brains. In the sagittal plane, $\mu$ DTI reveals the absence of the stria medullaris in the $\mathrm{Fz}^{-/-}$brain (Fig. $7 \mathrm{Q}, \mathrm{S}$ ), a defect that is difficult to appreciate with neurofilament staining because of the presence of neighboring fiber tracts (data not shown). In the false-color $\mu$ DTI image, the dorsoventral orientation of the stria medullaris readily distinguishes it from neighboring tracts.
Comparison of $\mu \mathrm{DTI}$ and neurofilament staining in the analysis of microscopic disorganization in the E18 brainstem In surveying the $\mathrm{Fz}^{-/-}$brain at E18 by neurofilament staining, we observed a widespread microscopic disorganization of fibers in the brainstem. At the level of the eighth nerve, the density of fibers in the WT and $\mathrm{Fz}^{-/-}$brainstems are similar, but the fiber orientations in the $\mathrm{Fz}^{-1-}$ sample show greater variability (Fig. $8 B, E)$. This microscopic disorganization appears to be below the resolution of $\mu \mathrm{DTI}$ in its current implementation (Fig. $8 C, F$ ).

\section{Visualizing defects in the $\mathrm{Fz}^{-/-}$brain using genetically directed neuronal markers}

As noted in Introduction, genetic techniques for sparse labeling of neurons have been available for several years. However, these approaches have not been widely applied to the analysis of mutant mice, perhaps in part because of the additional genetic crosses required to incorporate the reporter loci into the mutant background. To assess the utility of the CreER;ZAP system, we examined AP-stained vibratome sections of $\mathrm{WT}$ and $\mathrm{Fz}^{-/-}$ brains that were harvested at E18 after a single exposure to $4 \mathrm{HT}$ between E10 and E13. Because CreER coding sequences inserted at the ROSA26 locus are ubiquitously expressed, 4HT exposure at these ages leads to labeling of neurons, glia, and their precursors. With earlier times of 4HT exposure, labeled cells in the E18 brain are typically observed in small clusters, each of which represents 
the progeny of a single progenitor cell in which the Cre-mediated recombination occurred. The AP-labeled axons clearly delineate the major fiber tracts in the WT brain (Fig. 9A, $C, E$ ), including the internal capsule and corpus callosum, and reveal that these structures are absent and severely diminished, respectively, in the $\mathrm{Fz}^{-1-}$ brain (Fig. 9B,D,F). AP labeling also shows that most axons originating in the $\mathrm{Fz}^{-/-}$cortex do not project beyond the region immediately beneath the cortical pyramidal cells from which they arise (Fig. 9G,H). This last observation is of particular interest because neither MRI nor neurofilament staining can distinguish ascending from descending cortical fibers or the spatial trajectory of individual axons.

In the second genetically based labeling method, we selectively visualized the morphologies of cortical pyramidal cells using the Thy1-YFP line " $\mathrm{H}$ " developed by Feng et al. (2000), referred to hereafter as "Thy1-YFP." This line shows YFP fluorescence in several types of neurons, but, in the cortex, only pyramidal cells are labeled. In the postnatal brain, intense YFP fluorescence is seen in both pyramidal cell bodies and their axons. In contrast, in the E16 brain, only weak YFP fluorescence is seen in cell bodies (data not shown). Transgene expression at E18 presents an intermediate case, with sufficient YFP fluorescence to permit an analysis of both cell bodies and axonal projections in lightly fixed vibratome sections. Figure 10 compares the appearance of Thy1YFP-expressing pyramidal cell bodies and their axons in the WT and $\mathrm{Fz}^{-1-}$ cortex at E18. Although the density and location of pyramidal cell bodies are normal in the $\mathrm{Fz}^{-/-}$brain, the intermediate zone is filled with fragmented rather than contiguous processes.

It is likely that the fragmented material in the intermediate zone of the $\mathrm{Fz}^{-1-}$ brain represent the remnants of axons, and these are likely to be the light microscopic correlates of the numerous vesicle-like structures observed in this region by electron microcopy (Wang et al., 2002). The failure to visualize these fragments with anti-neurofilament staining most likely reflects their high density in the $\mathrm{Fz}^{-/-}$brain (in which case they are not readily resolved in the light microscope) and their low density in normal brains (in which case they are obscured by the large number of intact axons). Their presence is optimally revealed by the variegated labeling of cortical pyramidal cells from the Thy1-YFP transgene. At this stage, axonal fragmentation is not accompanied (or not yet accompanied) by a general demise of cortical pyramidal cells or other cortical neurons, because terminal deoxynucleotidyl transferase-mediated biotinylated UTP nick end labeling of fragmented DNA shows that cell death is barely detectable in the $\mathrm{Fz}^{-/-}$cortex at E18 (Wang et al., 2002).

\section{Spinal cord and peripheral nerve phenotypes in midgestation} $\mathrm{Fz3}^{-/-}$embryos

The analyses of the $\mathrm{Fz}^{-/-}$phenotype described above and reported by Wang et al. (2002) focused on rostral CNS defects in late gestation. To examine the possibility that additional defects
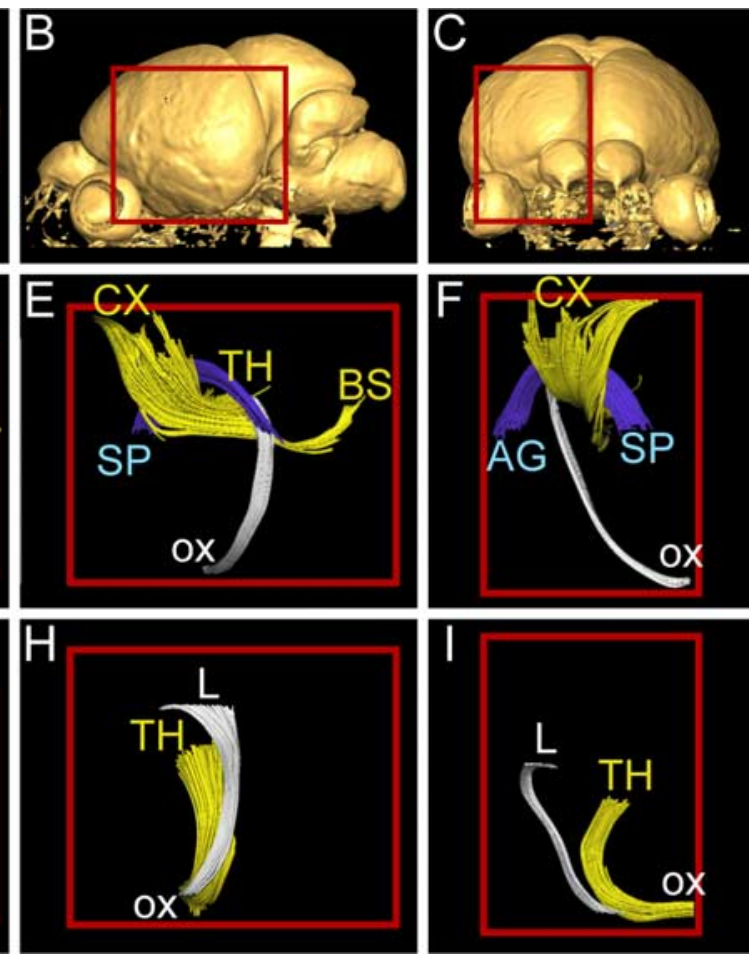

Figure 6. Three-dimensional reconstruction from $\mu$ DTI images of the major thalamic fiber tracts in WT and $F z 3^{-/-}$brains at E18. $\boldsymbol{A}$ C, Surface morphology of the WT brain showing with red squares the regions and orientations of the images in $\boldsymbol{D}-\boldsymbol{F}$. Matched images from an $\mathrm{Fz}^{-}{ }^{-}$brain are shown in $\mathbf{G}-\mathbf{I}$. In $\mathbf{D}-\mathbf{I}$, the optic tract is white; the cerebral peduncle and internal capsule (in WT; $\boldsymbol{D}-\boldsymbol{F}$ ) or the intrathalamic U-shaped fiber tract (in $\mathrm{Fz}^{-/-} ; \mathbf{G}-\mathbf{I}$ ) are yellow; and the stria terminalis is blue. AG, ic chiasm; SP, septum; TH, thalamus.

may manifest at earlier times in the spinal cord or peripheral nervous system (PNS), E12 embryos were examined by wholemount neurofilament staining (Fig. 11). In marked contrast to the development of rostral CNS axons, this analysis revealed a nearly normal pattern of axonal growth in the PNS, the only defect being a delay in the growth of the femoral nerve in $\mathrm{Fz}^{-/-}$ embryos. At the dorsal midline of the spinal cord, $\mathrm{Fz}^{-/-} \mathrm{em}-$ bryos exhibit a series of discrete neurofilament-rich zones (Fig. $11 D)$. In cross-sections, these are seen to consist of clusters of cells that appear to represent ectopic neurons and their associated axons (Fig. 11G,H). These clusters most likely arose from local defects in neural tube closure, suggesting that Fz3 also plays a role in directed cell migration.

\section{Discussion}

\section{Biology}

In the present study, we have defined a complex set of fiber tract defects in the $\mathrm{Fz}^{-1-}$ brain that confirms and extends our previous description of the $\mathrm{Fz}^{-/-}$mutant phenotype (Wang et al., 2002). From these analyses, we conclude that the following tracts are absent or greatly reduced in the $\mathrm{Fz}^{-/-}$brain: the anterior commissure, cerebral peduncle (corticospinal tract), corpus callosum, fornix, internal capsule (thalamocortical and corticothalamic tracts), stria medullaris, stria terminalis, and hippocampal commissure. Moreover, many longitudinal fibers in the brainstem appear to be disorganized. Fiber tracts that appear relatively unaltered in the $\mathrm{Fz}^{-/-}$brain include the fasciculus retroflexus, fimbriae, optic tract, mammillothalamic tract, hebenulopeduncular tract, and the trigeminal and optic nerves. Although the fasciculus retroflexus is present, its trajectory is altered, perhaps secondary to the absence of other tracts. The most unusual axon 

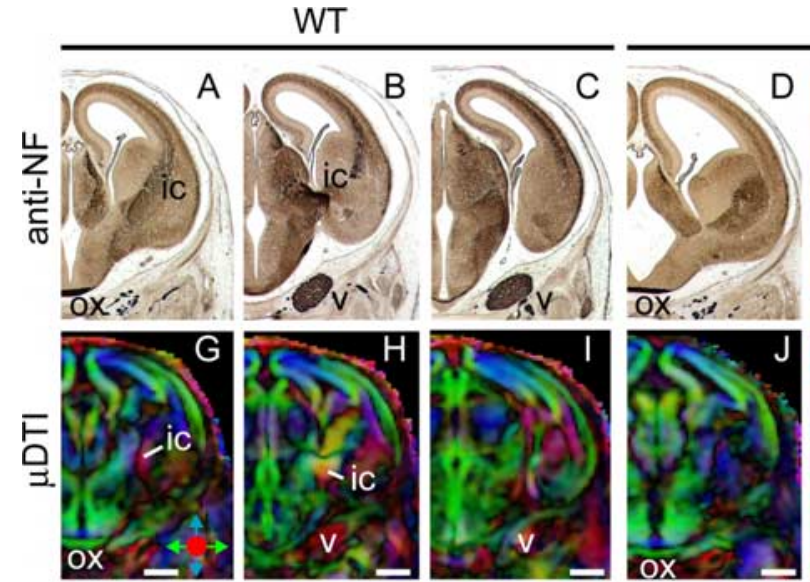

Fz3(-/-)
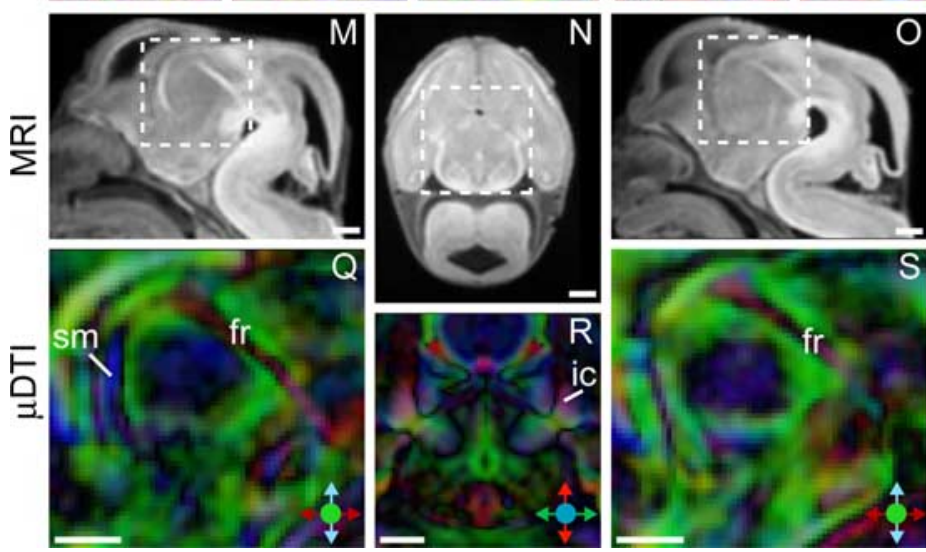

Figure 7. $\mathrm{F} Z 3^{-1-}$ fiber tract defects at $\mathrm{E} 14$ visualized by neurofilament immunostaining and $\mu \mathrm{DTI}$. $\boldsymbol{A}-\boldsymbol{L}$, Matched coronal sections beginning at the level of the optic chiasm $(\boldsymbol{A}, \boldsymbol{D}, \boldsymbol{G}, \boldsymbol{J})$ and $\sim 400 \mu \mathrm{m}(\boldsymbol{B}, \boldsymbol{E}, \boldsymbol{H}, \boldsymbol{K})$ or $\sim 800 \mu \mathrm{m}(\boldsymbol{C}, \boldsymbol{F}, \boldsymbol{I}, \boldsymbol{L})$ posterior to the optic chiasm. In $A-L$, the midline is adjacent to the left border of each image. The key in the bottom right of $G$ illustrates the false color code for oriented diffusion for $\mathbf{G}-\boldsymbol{L}$. $\boldsymbol{M}-\boldsymbol{P}$, Conventional MRI of the head shown by the square insets the locations of the enlarged $\mu \mathrm{DTl}$ images below (Q-T). $\boldsymbol{M}, \mathbf{O}, \mathbf{Q}, \mathbf{S}$, Sagittal views; $\boldsymbol{N}, \boldsymbol{P}, \boldsymbol{R}, \boldsymbol{I}$, horizontal views. The key in the bottom right of $\mathbf{Q}-\boldsymbol{T}$ shows the false color code for oriented diffusion for each of these panels. In the $\mathrm{Fz}^{3---}$ brain, the stria medullaris and the internal capsule are missing; the developing U-shaped fiber bundle is marked by an asterisk. fr, Fasciculus retroflexus; ic, internal capsule; sm, stria medullaris; ox, optic chiasm; V, trigeminal nucleus. Scale bars, $0.5 \mathrm{~mm}$.

tract anomaly in the $\mathrm{Fz}^{-1-}$ brain is the appearance of a novel $\mathrm{U}$-shaped connection between left and right thalamic nuclei, which may represent a default pathway for thalamocortical fibers that have failed to correctly target the cortex.

Interestingly, stalled or misrouted axons in the $\mathrm{Fz}^{-/-}$brain appear to fragment, as revealed by YFP imaging of cortical pyramidal cells. This fragmentation is reminiscent of the axonal fragmentation that follows axotomy of peripheral nerves, as visualized with the same Thy1-YFP transgene (Beirowski et al., 2004). In the developing CNS, fragmentation likely represents one stage in a normal process that eliminates aberrant axons. Like apoptosis, axonal fragmentation may occur at an appreciable rate during normal brain development but elude detection with conventional histologic methods. For example, fragmented axons might elude detection by carbocyanine dye tracing of fixed tissue because the dye will not diffuse across a membrane discontinuity. In general, fragmented axons would also be obscured by the far larger number of intact axons.

In contrast to the widespread defects observed in the $\mathrm{Fz}^{-/-}$ $\mathrm{CNS}$, the $\mathrm{Fz}^{-1-}$ PNS appears nearly normal, the only defect being the retarded growth of the femoral nerve. Finally, we describe clusters of neurons within the $\mathrm{Fz}^{-/-}$spinal cord that likely arise from errors in cell migration during neural tube closure, an hypothesis consistent with the curled tail phenotype seen

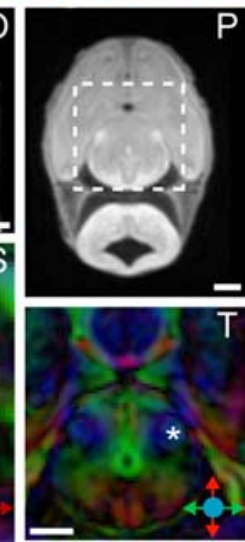

in all $\mathrm{Fz}^{-1-}$ embryos [an indication of an early neural tube defect (Peeters et al., 1998)] and our previous finding that several percentage of $\mathrm{Fz}^{-/-}$embryos exhibit an incompletely closed neural tube (Wang et al., 2002).

The constellation of $\mathrm{Fz}^{-/-}$fiber tract defects is strikingly similar to the defects recently reported in Celsr $3^{-/-}$mice (Tissir et al., 2005). In particular, Celsr $3^{-/-}$mice show a marked reduction or absence of the anterior commissure, cerebral peduncle, and internal capsule, as well as a disorganization of longitudinal fibers in the hindbrain tegmentum. Interestingly, in the Celsr $3^{-/-}$brain, a novel fiber bundle in the lateral thalamus follows an aberrant trajectory into the basal forebrain. Most likely, this aberrant bundle and the U-shaped intrathalamic bundle in the $\mathrm{Fz}^{-/-}$brain represent alternate trajectories for thalamic fibers that fail to project to the cortex via the internal capsule. This phenotypic difference suggests that additional Frizzled and/or Celsr family members may participate in thalamic axon growth and guidance such that the functions of Fz3 and Celsr3 are not completely coincident. It will be interesting to determine whether the tracts that are unaffected in the $F z 3^{-1-}$ brain use other Frizzled family members for growth and guidance or whether their development is independent of Frizzled signaling.

As noted by Tissir et al. (2005), the similarity between $\mathrm{Celsr}^{-1-}$ and $\mathrm{Fz}^{-1-}$ phenotypes suggests that these proteins function in the same signaling pathway. Celsr3 is one of three mammalian orthologs of Drosophila flamingo/starry night, a gene required for planar cell polarity (PCP) signaling in the adult cuticle and eye and for normal development of longitudinal axon fascicles in the embryonic nervous system (Chae et al., 1999; Usui et al., 1999). A second homolog, Celsr1, is required for neural tube closure and the correct orientation of stereociliary bundles in cochlear hair cells (Curtin et al., 2003). The members of the Flamingo family are all large integral membrane proteins, and Flamingo localization in the Drosophila wing is dependent on the presence of Frizzled, suggesting that the two proteins may be part of a complex at the cell surface. Together, these observations suggest that axonal growth and guidance mediated by Fz3 and Celsr3 use a PCP signaling system, and that Fz3 may also function in Celsr1dependent PCP signaling during neural tube closure.

\section{Technology}

The present study demonstrates the complementary strengths of $\mu \mathrm{DTI}$, neurofilament staining, and genetically directed cell marking in defining defects in mouse brain development. Of the three approaches, $\mu \mathrm{DTI}$ is the most useful in providing a global assessment of brain structure. Because $\mu$ DTI data are collected from the intact brain, it is free of sectioning artifacts and can be used for the quantitative analysis of shape and volume. In the current implementation of $\mu \mathrm{DTI}$, coronal, sagittal, and horizon- 

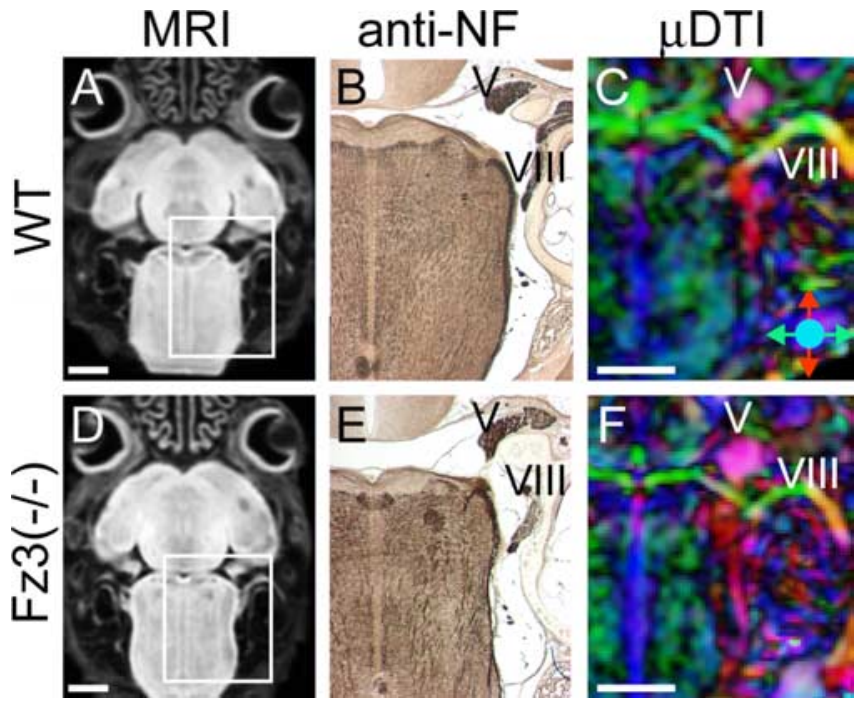

Figure 8. Local disorganization of fibers in the $\mathrm{Fz}^{-/-}$brainstem in horizontal sections at E18. $A, D$, Conventional MRI of the head showing with rectangular insets the locations of the enlarged $\mu \mathrm{DTI}$ images $(\boldsymbol{C}, \boldsymbol{F})$ and the matched neurofilament-stained sections $(\boldsymbol{B}, \boldsymbol{E})$. The midline is adjacent to the left border in $\boldsymbol{B}, \boldsymbol{C}, \boldsymbol{E}$, and $\boldsymbol{F}$. The key in the bottom right of $\boldsymbol{C}$ shows the false color code for oriented diffusion for each of these panels. The microscopic disorganization of the fiber bundles in the $\mathrm{Fz}_{2} \mathrm{3}^{-1-}$ brainstem $(\boldsymbol{E})$ are below the spatial resolution of $\mu \mathrm{DTI}(\boldsymbol{F}) . \mathrm{V}$, Trigeminal nerve; VIII, eighth nerve. Scale bars, $1 \mathrm{~mm}$.

tal sections are viewed simultaneously, the point at which the three views intersect is controlled by a cursor, and each plane of viewing can be arbitrarily tilted. These three features greatly facilitate the visualization of complex axonal trajectories and other three-dimensional structures.

In conventional MRI, individual white matter tracts cannot be distinguished and gray matter generates a relatively homogeneous signal. In contrast, $\mu$ DTI provides excellent definition of different fiber tracts based on differences in their orientation. Moreover, in some gray matter regions, such as the developing cortex, $\mu$ DTI reveals substantial information based on the relative weighting of oriented structures such as radial glia and dendritic processes. As cortical development proceeds, the relative weighting of radial versus tangential components changes in a characteristic manner (Zhang et al., 2003).

Neurofilament staining provides both a global assessment of fiber tract integrity as well as cellular or subcellular resolution, allowing the visualization of subtle defects in fiber organization or fasciculation that are below the spatial resolution of $\mu \mathrm{DTI}$. Whole-mount neurofilament staining provides excellent visualization of the developing peripheral nervous system, nearly all of which is below the resolution of $\mu$ DTI. However, neurofilament staining does not easily resolve adjacent or interdigitated fiber tracts, a major limitation in identifying defects in smaller CNS tracts (e.g., the stria medullaris at E14). Additionally, like all classic histologic methods, neurofilament staining suffers from the constraint that each brain can be viewed only in one plane of section, and that the dataset of stained sections cannot be easily manipulated to reveal complex three-dimensional relationships.

In mice, the development of genetically directed neuronal marking methods is still in its infancy. The Thy1-YFP transgenic line used here represents the technically simplest approach: the temporal and spatial patterns of reporter gene expression and the density of expressing cells are determined by the random integration site of the transgene (Feng et al., 2000). In some Thy1-YFP lines, transgene expression is sufficiently sparse that the mor-
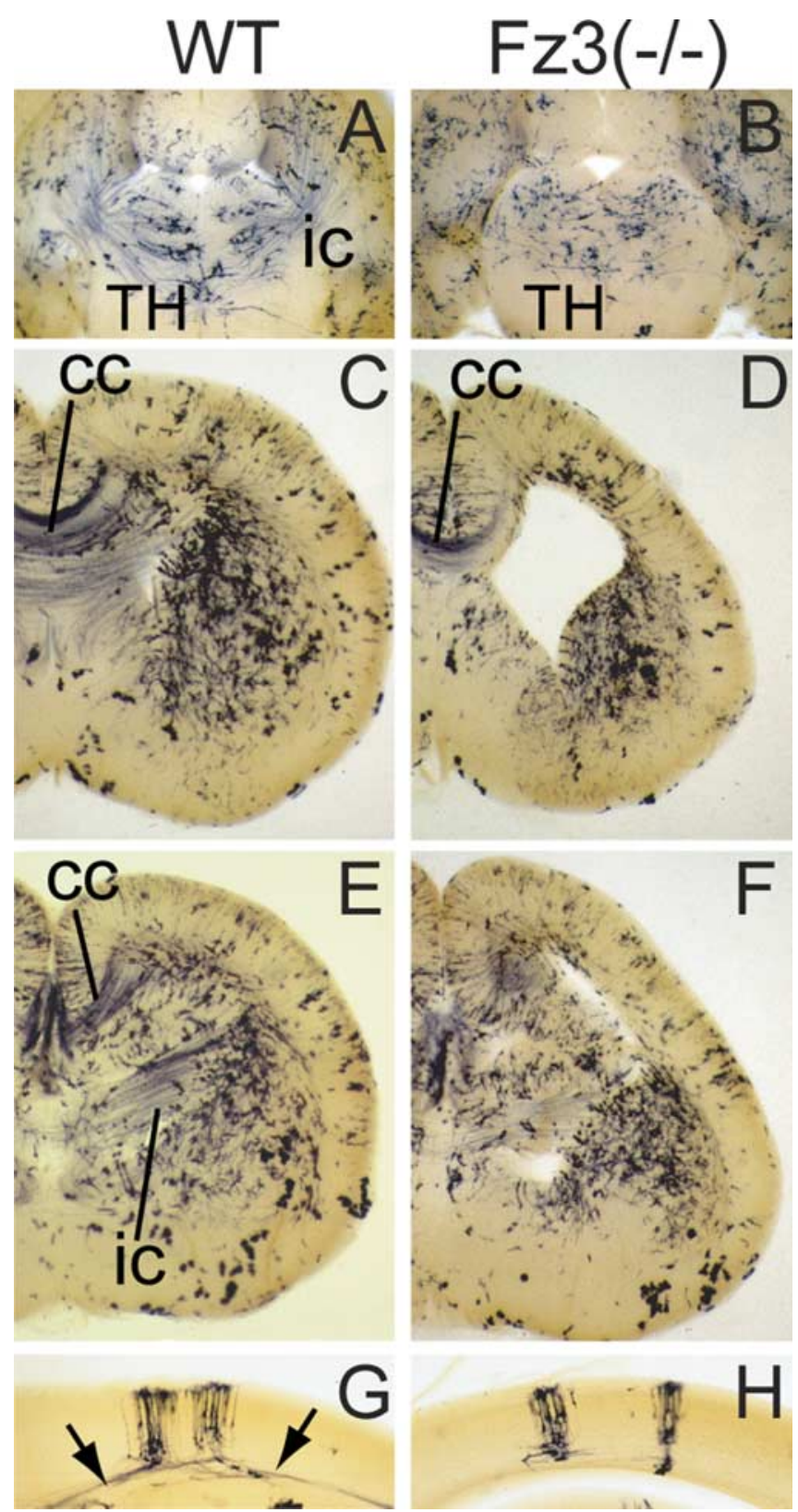

Figure 9. Defects in $\mathrm{F}_{3} 3^{-/-}$fiber tracts visualized with sparse AP labeling of neurons in brains of WT and $F_{Z 3}{ }^{-/-}$littermates at E18. Littermates carrying the ROSA26-CreER and ZAP transgenes were exposed to a single maternal injection of $200 \mu \mathrm{g}$ of $4 \mathrm{HT}$ on gestational days $\mathrm{E} 12(\boldsymbol{A}, \boldsymbol{B}), \mathrm{E} 13(\boldsymbol{C} \boldsymbol{-})$, or E10 $(\boldsymbol{G}, \boldsymbol{H}) \cdot \boldsymbol{A}, \boldsymbol{B}$, Horizontal sections with the thalamus in the center. The fibers of the left and right internal capsules are seen in the WT brain but are absent from the $\mathrm{FZ}_{2}{ }^{-1-}$ brain. $\mathbf{C}-\boldsymbol{F}$, Coronal sections showing the smaller corpus callosum and the absence of the internal capsule in the $\mathrm{F}_{2} 3^{-/-}$brain. $\boldsymbol{G}, \boldsymbol{H}$, Two small clusters of labeled cortical neurons show extensive axonal projections (arrows) in WT but not $\mathrm{Fz}^{-/-}$. In $C-F$, the midline is adjacent to the left border of each image. cc, Corpus callosum; ic, internal capsule; $T H$, thalamus.

phologies of individual neurons can be resolved and monitored in living tissue (Trachtenberg et al., 2002). The diversity of expression patterns observed thus far with random integration of the Thyl promotor suggests that many additional expression patterns could be generated by random integration of other promoters or combinations of promotor elements.

The second genetic marking method used here combines a ubiquitously expressed CreER recombinase, a Cre-activated AP reporter, and low-dose $4 \mathrm{HT}$ injection to generate sparse labeling of neurons (Badea et al., 2003). By preparing coronal sections of 

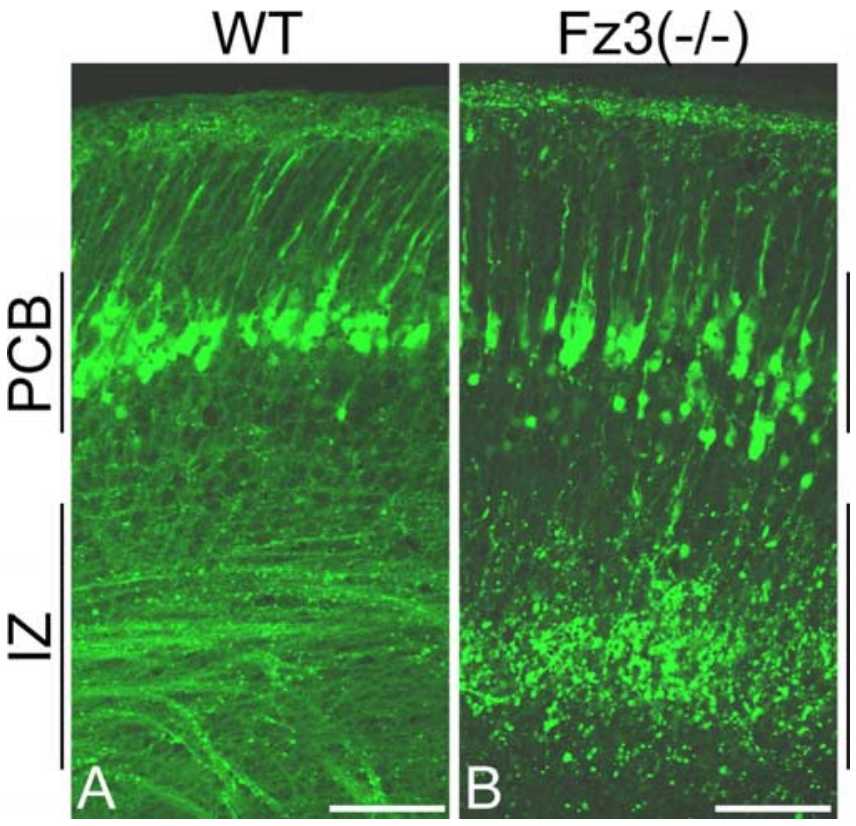

Figure 10. Cortical pyramidal cells and fragmented intermediate zone axons in the $\mathrm{Fz}_{3}{ }^{-1}$ brain visualized with a Thy1-YFP transgene at E18. Coronal sections from littermates carrying the Thy1-YFP transgene were imaged by confocal microscopy. The surface of the cortex is at the top, the pyramidal cell bodies (PCB) are in the center, and the intermediate zone (IZ) is near the bottom of the image. From an examination of multiple vibratome sections in different focal planes, it is clear that the fluorescent objects in the intermediate zone of the $\mathrm{F}_{2} 3^{-/-}$cortex are also discontinuous in the dimension perpendicular to the image shown here. Scale bars, $100 \mu \mathrm{m}$.

an E18 head at a thickness of $300 \mu \mathrm{m}$, a single late gestation brain can be analyzed with $\sim 20$ sections. The three-dimensional trajectory of each axon is readily appreciated because the thick tissue sections can be rendered optically clear in benzyl benzoate/benzyl alcohol. With the labeling densities used here, the major fiber tracts are represented by several dozen axons with parallel trajectories. We note that this density of axonal labeling is too low to reliably identify smaller tracts.

In applying the CreER;ZAP method to the developing brain, we initiated 4HT-dependent Cre-mediated recombination several days before tissue processing to allow sufficient time for the accumulation of the AP reporter. When 4HT exposure occurs during the period of active neuronal proliferation (before approximately E15 for cerebral cortical neurons), AP activity is seen in a small clusters of cells, with each cluster representing the clonal progeny of individual precursors in which a recombination event occurred. Because the number of initiating events is determined by $4 \mathrm{HT}$ dose and the size of the resulting clones is determined by the timing of $4 \mathrm{HT}$ exposure (with earlier exposure leading to larger clones) (Badea et al., 2003), one can readily produce a brain in which many small clusters of labeled neurons efficiently sample the major structures.

It is interesting to compare the two genetic methods for visualizing neurons with the more widely used method of axon tracing with carbocyanine dyes (e.g., DiI). Each of these methods has the virtue that cell labeling is independent of the abundance, diversity, or subcellular localization of marker proteins, constraints that severely limit immunohistochemical marking methods. In carbocyanine dye tracing, a small crystal or aliquot of concentrated tracer is placed at a defined location, and the experimenter subsequently observes the diffusion of the tracer in both anterograde and retrograde directions. This method suffers from two technical limitations: (1) neurons close to the labeling site are

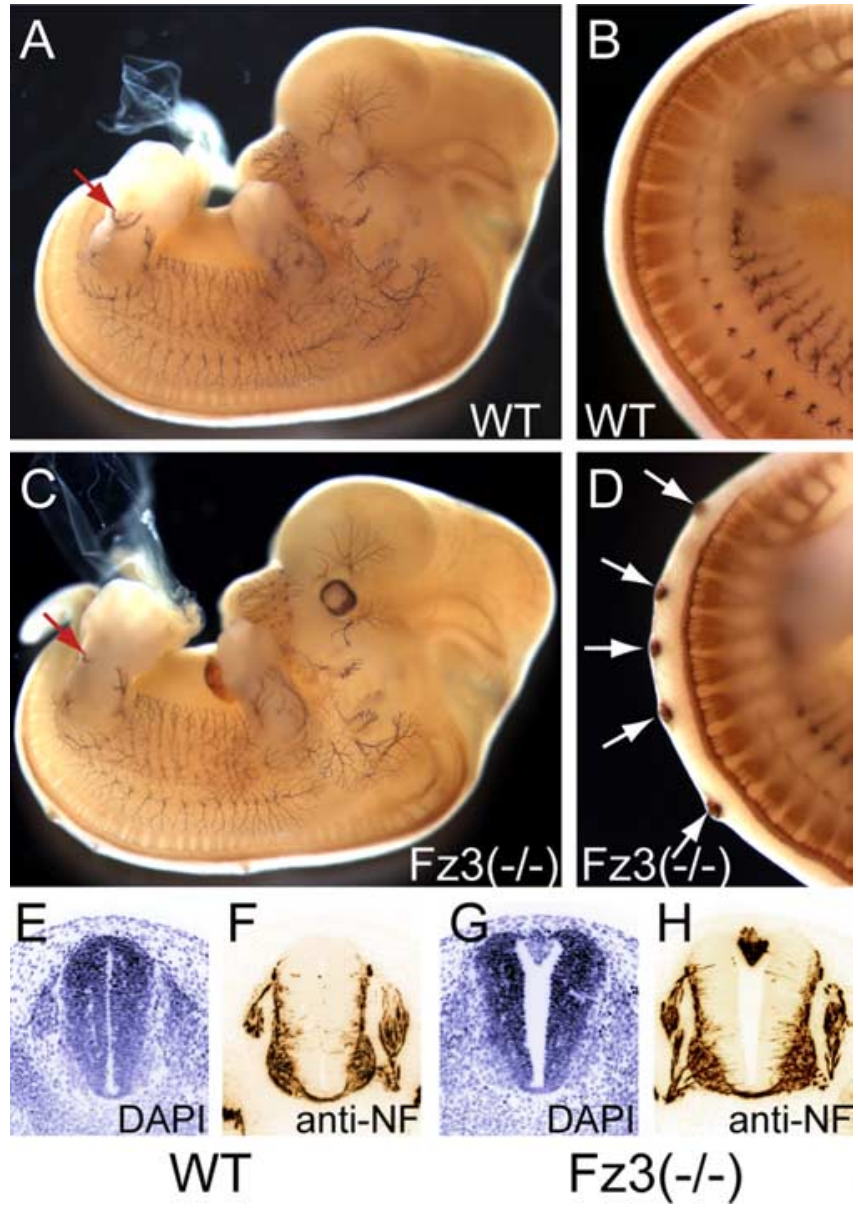

Figure 11. Intermittent defects in spinal cord morphology but nearly normal peripheral nerve development in $\mathrm{E}_{12} \mathrm{Fz}^{-1-}$ embryos. A, C, Whole-mount neurofilament staining shows that, except for delayed outgrowth of the femoral nerve (red arrows), the development of the $\mathrm{Fz}^{-1-}$ peripheral nervous system is indistinguishable from WT. $B, D, A$ series of discrete neurofilament-rich zones (arrows) are present along the length of the $\mathrm{Fz}_{2}{ }^{-1-}$ spinal cord. $\boldsymbol{E}-\boldsymbol{H}$, Cross-sections of WT and $\mathrm{Fz}^{-1-}$ spinal cords stained with $4^{\prime}, 6^{\prime}$-diamidino-2phenylindole (DAPI) or stained for neurofilament (NF). The $\mathrm{Fz}^{-/-}$spinal cord sections pass through one of the neurofilament-rich zones and show clusters of cells that likely represent ectopic neurons and their associated axons.

obscured by the intense fluorescence of residual tracer; and (2) any manipulation that disrupts membrane integrity causes the tracer to diffuse nonspecifically, a limitation that precludes cutting thin sections or clarifying thick sections with organic solvents. Despite these drawbacks, carbocyanine dye tracing is widely used because it is technically simple and it produces intense labeling of individual axons and dendrites.

Compared with carbocyanine dye tracing, genetic labeling methods have several favorable characteristics: (1) both deep and superficial neurons are accessible to labeling without the mechanical disruption that accompanies tracer placement, (2) no parts of the tissue are obscured by the labeling method, (3) in the case of AP histochemistry, the tissue can be rendered optically clear without loss of the label, and (4) the potential exists to direct the labeling to defined subsets of neurons based on the specificity of reporter gene expression. At present, the last of these has only been imperfectly realized: in this study, the identities of the genetically labeled neurons were determined either by the random site of transgene integration (Thyl-YFP) or stochastically within each labeled brain (CreER;ZAP). Future refinements in this technology, including the generation of knock-in mice with cell-type- 
specific reporter expression, should lead to progressively greater temporal and spatial control over cell marking.

\section{References}

Badea TC, Wang Y, Nathans J (2003) A noninvasive genetic/pharmacologic strategy for visualizing cell morphology and clonal relationships in the mouse. J Neurosci 23:2314-2322.

Basser PJ, Mattiello J, LeBihan D (1994) MR diffusion tensor spectroscopy and imaging. Biophys J 66:259-267.

Beirowski B, Berek L, Adalbert R, Wagner D, Grumme DS, Addicks K, Ribchester RR, Coleman MP (2004) Quantitative and qualitative analysis of Wallerian degeneration using restricted axonal labelling in YFP-H mice. J Neurosci Methods 134:23-35.

Chae J, Kim MJ, Goo JH, Collier S, Gubb D, Charlton J, Adler PN, Park WJ (1999) The Drosophila tissue polarity gene starry night encodes a member of the protocadherin family. Development 126:5421-5429.

Curtin JA, Quint E, Tsipouri V, Arkell RM, Cattanach B, Copp AJ, Henderson DJ, Spurr N, Stanier P, Fisher EM, Nolan PM, Steel KP, Brown SD, Gray IC, Murdoch JN (2003) Mutation of Celsrl disrupts planar polarity of inner ear hair cells and causes severe neural tube defects in the mouse. Curr Biol 13:1129-1133.

Feng G, Mellor RH, Bernstein M, Keller-Peck C, Nguyen QT, Wallace M, Nerbonne JM, Lichtman JW, Sanes JR (2000) Imaging neuronal subsets in transgenic mice expressing multiple spectral variants of GFP. Neuron 28:41-51.

Guo N, Hawkins C, Nathans J (2004) Frizzled6 controls hair patterning in mice. Proc Natl Acad Sci USA 101:9277-9281.

Haugland R (2002) Handbook of fluorescent probes and research products, Ed 9. Eugene, OR: Molecular Probes.

Jacobs RE, Ahrens ET, Meade TJ, Fraser SE (1999) Looking deeper into vertebrate development. Trends Cell Biol 9:73-76.

Lobe CG, Koop KE, Kreppner W, Lomeli H, Gertsenstein M, Nagy A (1999) $\mathrm{Z} / \mathrm{AP}$, a double reporter for cre-mediated recombination. Dev Biol 208:281-292.

Lyuksyutova AI, Lu CC, Milanesio N, King LA, Guo N, Wang Y, Nathans J, Tessier-Lavigne M, Zou Y (2003) Anterior-posterior guidance of commissural axons by Wnt-frizzled signaling. Science 302:1984-1988.

Mori S, van Zijl PC (1998) A motion correction scheme by twin-echo nav- igation for diffusion-weighted magnetic resonance imaging with multiple RF echo acquisition. Magn Reson Med 40:511-516.

Mori S, Itoh R, Zhang J, Kaufmann WE, van Zijl PC, Solaiyappan M Yarowsky P (2001) Diffusion tensor imaging of the developing mouse brain. Magn Reson Med 46:18-23.

Nauta WJ, Feirtag M (1986) Fundamental neuroanatomy, pp 50-61. New York: Freeman.

Peeters MC, Schutte B, Lenders MH, Hekking JW, Drukker J, Van Straaten HW (1998) Role of differential cell proliferation in the tail bud in aberrant mouse neurulation. Dev Dyn 211:382-389.

Tissir F, Bar I, Jossin Y, Goffinet AM (2005) Protocadherin Celsr3 is crucial in axonal tract development. Nat Neurosci 8:451-457.

Trachtenberg JT, Chen BE, Knott GW, Feng G, Sanes JR, Welker E, Svoboda $\mathrm{K}$ (2002) Long-term in vivo imaging of experience-dependent synaptic plasticity in adult cortex [abstract]. Nature 420:788-794.

Usui T, Shima Y, Shimada Y, Hirano S, Burgess RW, Schwarz TL, Takeichi M, Uemura T (1999) Flamingo, a seven-pass transmembrane cadherin, regulates planar cell polarity under the control of Frizzled. Cell 98:585-595.

Wang Y, Huso D, Cahill H, Ryugo D, Nathans J (2001) Progressive cerebellar, auditory, and esophageal dysfunction caused by targeted disruption of the frizzled-4 gene. J Neurosci 21:4761-4771.

Wang Y, Thekdi N, Smallwood PM, Macke JP, Nathans J (2002) Frizzled-3 is required for the development of major fiber tracts in the rostral CNS J Neurosci 22:8563-8573.

Westin CF, Maier SE, Mamata H, Nabavi A, Jolesz FA, Kikinis R (2002) Processing and visualization for diffusion tensor MRI. Med Image Anal 6:93-108.

Woods RP, Grafton ST, Watson JDG, Sicotte NL, Mazziotta JC (1998) Intersubject validation of linear and nonlinear models. J Comput Assist Tomogr 22:153-165.

Zhang J, Richards LJ, Yarowsky P, Huang H, van Zijl PC, Mori S (2003) Three-dimensional anatomical characterization of the developing mouse brain by diffusion tensor microimaging. NeuroImage 20:1639-1648

Zhao C, Aviles C, Abel RA, Almli CR, McQuillen P, Pleasure SJ (2005) Hippocampal and visuospatial learning defects in mice with a deletion of frizzled 9, a gene in the Williams syndrome deletion interval. Development 132:2917-2927. 